Morga MANZANARES, R. y SERRANo-MARTínEZ, C.., «Visiones y experiencias familiares y profesionales en la prevención de la institucionalización de las personas con discapacidad intelectual en La Rioja», REDUR 17, diciembre 2019, págs. $167-180$. ISSN 1695-078X. doi: 10.18172/redur.4398

\title{
VISIONES Y EXPERIENCIAS FAMILIARES Y PROFESIONALES EN LA PREVENCIÓN DE LA INSTITUCIONALIZACIÓN DE LAS PERSONAS CON DISCAPACIDAD INTELECTUAL EN LA RIOJA
}

\author{
RUTH MORGA MANZANARES \\ Trabajadora Social del Centro de Atención Diurna La Sierra (La Rioja) \\ ASPRODEMA \\ ruthmorga@asprodema.org
}

\section{CECILIA SERRANO-MARTÍNEZ}

PROFESORA CONTRATADA INTERINA

UNIVERSIDAD DE LA RIOJA

cecilia.serrano@unirioja.es

SUMARIO: I. INTRODUCCIÓN. II. EL MODELO BIO-PSICO-SOCIAL DE DISCAPACIDAD Y LA DiSCAPACIDAD INTELECTUAL. III. INSTITUCIONALIZACIÓN COMO MODELO DE ATENCIÓN RESIDENCIAL. IV. OBJETIVOS Y METODOLOGÍA DEL ESTUDIO. V. MOTIVOS PARA SOLICITAR PLAZA DE RESIDENCIA Y BUENAS PRÁCTICAS FAMILIARES. VI. REFLEXIONES PARA LA PREVENCIÓN DE LA INSTITUCIONALIZACIÓN. VII. CONCLUSIONES. VIII. BIBLIOGRAFÍA.

RESUMEN: Un recurso para el cuidado y atención de las personas con discapacidad intelectual es la institucionalización, la cual incluye a las personas con necesidades de apoyo en una institución dirigida a atender y dar respuestas a sus necesidades. Un elemento para prevenir esta tendencia y buscar otras alternativas es conocer las visiones de los propios familiares y de los profesionales que trabajan directamente con el colectivo. El presente artículo ofrece estos discursos, a través de la realización de dos Grupos de Discusión. Entre las conclusiones principales, se deben realizar prácticas que favorezcan el mantenimiento las relaciones familiares y que faciliten el establecimiento de relaciones sociales con otras personas sin discapacidad en entornos inclusivos. Las personas residentes deben ejercer su autodeterminación en el día a día de la vivienda, tomando decisiones acerca de sus asuntos cotidianos.

Palabras Clave: Discapacidad intelectual, institucionalización, Grupos de Discusión, Trabajo Social. 


\section{VISIONS AND PROFESSIONAL EXPERIENCES IN THE PREVENTION OF THE INSTITUTIONALIZATION OF PERSONS WITH INTELLECTUAL DISABILITIES IN LA RIOJA}

ABSTRACT: A resource for take care and attention of people with intellectual disabilities is the institutionalization which includes includes people with support needs in an institution aimed at serving and responding to their needs. An element to prevent this trend and find other alternatives is to know the visions of family members and professionals who work directly with that group of people. This article offers these speeches, through the realization of two Discussion Groups. Among the main conclusions, practices that favour the maintenance of family relationships and that facilitate the establishment of social relationships with other people without disabilities in inclusive environments should be carried out. Residents must apply their self-determination in the day-to-day living, making decisions about their everyday matters.

KEYWORDS: Intellectual disabilities, institutionalization, Discussion Group, Social Work.

\section{Introducción}

Uno de los recursos para el cuidado y atención de las personas con discapacidad intelectual (DI) ha sido históricamente, y continúa siendo, la institucionalización, la cual incluye a las personas con necesidades de apoyo en una institución dirigida a atender y dar respuesta a sus necesidades. En Europa, a raíz de la aprobación de la Convención Internacional de los Derechos de las Personas con Discapacidad ONU (2006), se están revisando los modelos de los servicios de atención a las personas con discapacidad. Se está tendiendo hacia modelos normalizados y de inclusión social. La desinstitucionalización de las personas con discapacidad atendidas en instituciones forma parte de las estrategias y políticas de los diferentes países de la Unión Europea. Un medio para prevenir la tendencia de la institucionalización y buscar otras alternativas es conociendo las visiones de los propios familiares y de los profesionales que trabajan directamente con el colectivo.

El presente artículo pretende ofrecer los resultados extraídos de los discursos de familiares y profesionales; así como conocer los motivos que han impulsado a las familias a solicitar plaza de residencia, las buenas prácticas para mantener las relaciones con sus familiares residentes, y conocer la opinión de profesionales del sector acerca de qué medidas se podrían impulsar en pro del diseño de servicios residenciales enfocados al fomento de las relaciones sociales y familiares. La metodología aplicada ha sido la realización de dos Grupos de Discusión, uno a profesionales y otro a familiares de personas con discapacidad intelectual de la Comunidad Autónoma de La Rioja.

Entre las principales conclusiones alcanzadas cabe destacar que, para poner en práctica servicios residenciales sin caer en la institucionalización, se deben realizar prácticas que favorezcan el mantenimiento las relaciones familiares y que faciliten el establecimiento de relaciones sociales con otras personas sin discapacidad en entornos inclusivos. Por otra parte, las personas residentes deben ejercer su autodeterminación en el día a día de la vivienda, de manera práctica y real, tomando decisiones acerca de los asuntos más cotidianos. Del mimo modo, se vislumbra que la disciplina del Trabajo Social posee herramientas aplicadas que permiten apoyar a las personas con discapacidad 
intelectual e intervenir desde lo individual y familiar hasta lo grupal y comunitario; ofreciendo calidad y favoreciendo que mejoren en las diferentes dimensiones de su vida.

\section{El Modelo bio-psico-social de Discapacidad y la Discapacidad Intelectual}

El modelo actual de discapacidad de referencia internacional es el llamado modelo bio-psico-social descrito en la Clasificación Internacional del Funcionamiento, la Discapacidad y de la Salud (CIF) publicado por la Organización Mundial de la Salud en el año 2001. Uno de los objetivos que persigue esta clasificación es "establecer un lenguaje común para describir la salud y los estados relacionados con ella" y señala que no se refiere solo a personas con discapacidad, ya que "tiene una aplicación universal" (OMS, 2001).

En cuanto al ámbito, la CIF define dos componentes claves para entender la clasificación: el Componente de Funcionamiento y Discapacidad y el Componente de Factores Contextuales que incluyen tanto los factores personales como los ambientales. El funcionamiento y la discapacidad de una persona se concibe como una interacción dinámica entre los estados de salud (enfermedades, trastornos, lesiones, traumas, etc.) y los factores contextuales.

En el funcionamiento humano intervienen las funciones que cumplen los diferentes sistemas del cuerpo humano, y las estructuras corporales que los componen, las actividades que realiza la persona en su vida cotidiana, y la participación en situaciones que vive la persona. Este funcionamiento está mediado por los factores ambientales (escenarios físicos, sociales y actitudinales del entorno) y personales.

En cuanto a la discapacidad intelectual, la Asociación Americana de Discapacidades Intelectuales y del Desarrollo (AAIDD); institución de referencia a nivel internacional en el ámbito científico y técnico; define y clasifica la discapacidad intelectual y señala cómo se han de prestar apoyos a este grupo de población. La AAIDD indica que la discapacidad intelectual "se caracteriza por limitaciones significativas tanto en el funcionamiento intelectual como en la conducta adaptativa, tal y como se ha manifestado en habilidades adaptativas conceptuales, sociales y prácticas. Esta discapacidad aparece antes de los 18 años" (Verdugo et al., 2011, p. 33). Para clarificar esta definición, resulta necesario incluir una serie de premisas que faciliten una aplicación operativa de discapacidad intelectual (Verdugo, 2010). Estas premisas (Schalock, 2009) son las siguientes:

- Las limitaciones en el funcionamiento presente se deben considerar en el contexto de ambientes comunitarios típicos de los iguales en edad y cultura.

- Una evaluación válida tiene en cuenta la diversidad cultural y lingüística, así como las diferencias en comunicación y en aspectos sensoriales, motores y conductuales.

- En una persona las limitaciones coexisten habitualmente con capacidades.

- Un propósito importante de la descripción de limitaciones es el desarrollo de un perfil de necesidades de apoyo.

- Si se mantienen apoyos personalizados apropiados durante un largo periodo, el funcionamiento en la vida de la persona con Discapacidad Intelectual generalmente mejorará. 
La definición anterior se puede relacionar con el concepto de "funcionamiento humano" descrito en la definición de discapacidad de la CIF. En este sentido, la AAIDD "ve la discapacidad como el ajuste entre las capacidades de la persona y el contexto en el que esta funciona" (Verdugo et al., 2011, p. 42). La DI se refiere a un estado de funcionamiento y no es en sí una condición de la persona.

\section{Institucionalización como modelo de atención residencial}

El término de institucionalización se traduce como la acción de incluir a una persona con necesidades de apoyo en una institución, como servicio para atender estas necesidades. Huete García et al. (2015, p. 15) lo definen como "el ingreso, voluntario o involuntario, en una instalación que presta servicios integrales, y que precisan la residencia de la persona con discapacidad, de manera permanente o temporal de larga duración”. Históricamente, las instituciones han sido el modelo principal de atención residencial y de atención para diferentes colectivos con necesidades de apoyo; incluidos entre ellos las personas con discapacidad intelectual (Huete García, Huete García y Martín Gómez, 2015). En Europa, después de la Segunda Guerra Mundial, comienza a producirse un paulatino cambio de modelo y se produce en algunos países un proceso de desinstitucionalización, principalmente en instituciones psiquiátricas. Las necesidades de apoyo de sus beneficiarios pasan a ser atendidas en entornos comunitarios. Los motivos de este cambio de modelo tienen que ver con cambios ideológicos, escándalos relacionados con las instituciones, creación de organizaciones sociales promovidas, en ocasiones, por las propias personas afectadas o sus familiares, y por el análisis de estas instituciones desde el punto de vista de sus costes económicos y de los beneficios que obtenían las personas atendidas en las mismas (Huete, García et al., 2015).

En Europa, a raíz de la aprobación de la Convención Internacional de los Derechos de las Personas con Discapacidad ONU (2006), se están revisando los modelos de los servicios de atención a las personas con discapacidad. Se está tendiendo hacia modelos normalizados y de inclusión social. La desinstitucionalización de las personas con discapacidad atendidas en instituciones forma parte de las estrategias y políticas de los diferentes países de la Unión Europea.

Recientemente, el Comité sobre los Derechos de las Personas con Discapacidad de la ONU (2019) en su último informe de seguimiento de la aplicación en España de la Convención Internacional de los Derechos de las Personas con Discapacidad, se refiere al Derecho a vivir de forma independiente y a ser incluido en la comunidad. Este mismo informe insta al Gobierno de España a suprimir los fondos públicos destinados a la construcción de instituciones residenciales, y a que destine esos fondos al desarrollo de una estrategia de desinstitucionalización y en servicios para personas con discapacidad de vida independiente en la comunidad y de asistencia personal. En palabras de la Coalición Europea para la Vida Comunitaria, "cualquier lugar en el que las personas son aisladas, separadas y/o obligadas a vivir juntos. Una institución es también cualquier lugar en el que las personas no tienen, o no se les permite, ejercer un control sobre su vida y sus decisiones del día a día" (Huete García et al., 2015, p. 9). En relación con esta definición, conviene revisar el concepto de "institución total" de Erving Goffman (1961, citado por Huete García 
et al., 2015, p. 9), que describe a las instituciones como lugares donde toda la vida de la persona está organizada y dirigida por una misma autoridad (en el caso de las instituciones para personas con discapacidad, la dirección y los profesionales), se desarrolla en continua compañía de otras personas (principalmente con las mismas características y necesidades de apoyo), y todas las actividades de la vida diaria de las personas que viven en ellas están programadas, además de establecidas por el sistema de normas de la institución (a qué hora se acuestan, cuando se duchan, horario y días para recibir llamadas de teléfono de familiares, etc.).

En la publicación "Institucionalización y dependencia” del Instituto de Mayores y Servicios Sociales (2006, p. 14) se hace referencia a diferentes efectos adversos de la institucionalización para las personas que viven en centros. Uno de ellos es la perdida de contacto con la realidad por la falta de responsabilidades y de control en los aspectos de la vida cotidiana, realidad grupal y no individual, establecimiento de clases sociales entre los propios residentes en función de sus niveles de autonomía, de intensidad de necesidades de apoyo, o de sus discapacidades. Por otro lado, también se da un alto grado de arbitrariedad institucional en la atención a las personas, en el sentido de que aspectos personales de la vida de las personas los determina la institución en base a las plantillas de personal de atención directa y a horarios concretos ajenos a los deseos de los residentes, la negación o restricción de aspectos emocionales o sexuales de las personas que viven en una institución, y la existencia de errores. Éstos tienen consecuencias en la calidad de la atención y en la persona atendida, por la aplicación de protocolos referidos a realidades colectivas y que no tienen en cuenta las realidades individuales. Otra de las claras consecuencias de la estancia en una institución es la dificultad y/o restricción de las relaciones entre la persona que vive en ella y su entorno familiar y social.

En cuanto a volúmenes y datos, teniendo en cuenta la Encuesta sobre Discapacidad, Autonomía personal y situaciones de Dependencia (EDAD, 2008) se estima que la población con discapacidad en España es de 4,12 millones de personas, siendo un 6,5\% del total de personas con discapacidad el grupo de personas que vive en instituciones. Este dato apunta a que, aunque en España un número importante de personas viven en instituciones y por tanto esta realidad merece una atención especial, el modelo de atención a personas con discapacidad tiene una base fundamental en el hogar y en la familia como principal fuente de apoyos. Teniendo en cuenta la edad de los residentes, el mayor grupo de personas que vive en instituciones es el de aquellas que tienen 65 años o más $(82,6 \%$ del total de residentes). Esta alta proporción de residentes mayores de 65 años es debido a la mayor oferta de servicios residenciales a partir de esa edad y al aumento de la prevalencia de las situaciones de discapacidad/ dependencia asociadas al proceso de envejecimiento en el conjunto de la población a partir de esa edad.

Por otro lado, hay un grupo de 46.879 personas residentes en centros en edad laboral, lo que permite afirmar que este grupo de población tiene asociada una discapacidad de causa congénita o sobrevenida por diferentes causas. Teniendo en cuenta la deficiencia que ha causado esa discapacidad en datos de la EDAD-centros se puede comprobar que el grupo de residentes en edad laboral, principalmente tiene alguna deficiencia mental (87,36\% de ese grupo de personas). En el informe elaborado por Huete García et al. (2015) 
constatan que el $90 \%$ de las personas que viven en centros de atención a personas con discapacidad tienen discapacidad intelectual y/o enfermedad mental.

En cuanto al impacto de la institucionalización en las relaciones familiares y/o sociales, cabe indicar que supone una pérdida de oportunidades en el plano social, en cuanto a participación y establecimiento de relaciones sociales, y se traduce en una pérdida de contacto con su realidad social y en consecuencia el aislamiento de la persona (Huete García et al., 2015). Entre esas relaciones sociales que se ven dificultadas se incluyen las relaciones con la familia y el entorno. Sin embargo, es necesario constatar que la participación y relaciones sociales, junto con las oportunidades de autonomía personal, son aspectos que permiten la mejora de la calidad de vida de las personas que viven en una institución.

El internamiento de una persona con discapacidad en un centro suele comenzar siendo una solución temporal o "de respiro" para la familia, que se ve desbordada por la tarea de cuidado que supone atender las necesidades de su familiar con discapacidad. Cabe señalar que, con el paso del tiempo, el centro pasa de ser una solución temporal, de último recurso, a una de larga estancia que suele tener como consecuencia la "desconexión" paulatina de la persona con discapacidad y su familia (Huete García et al., 2015, p. 59). No obstante, en ocasiones las personas con discapacidad, especialmente aquellas con necesidades de apoyo complejas, al ir a vivir a una residencia ven mejorada su calidad de vida, sobre todo en aspectos relacionados con la salud, y en los casos de personas que han vivido situaciones de abandono o condiciones de vida muy precarias (Huete García et al., 2015, p. 60).

\section{Objetivos y metodología del estudio}

El presente artículo pretende ofrecer los resultados extraídos de los discursos de familiares y profesionales, en relación a la institucionalización de las personas con discapacidad intelectual en la Comunidad Autónoma de La Rioja. Para ello, se establecen una serie de objetivos específicos, que son los siguientes:

- Conocer los motivos que han impulsado a las familias a solicitar plaza de residencia y las buenas prácticas que familiares para el mantenimiento de sus relaciones con sus familiares residentes.

- Conocer la opinión de profesionales del sector acerca de qué medidas se pueden impulsar en el diseño de servicios residenciales enfocados al fomento de las relaciones sociales y familiares.

Para llevar a cabo la investigación se ha utilizado la técnica cualitativa del Grupo de Discusión. Se han realizado dos Grupos de Discusión, uno a profesionales que trabajan con personas con discapacidad y otro a familiares de personas con discapacidad intelectual (en adelante PDI) que ocupan plaza o están en lista de espera para obtener una residencia.

- Grupo de Discusión de Profesionales (en adelante GP): Se ha contado con seis profesionales con una larga experiencia en el trabajo con PDI. Éstas han sido: tres trabajadoras sociales de entidades que trabajan con PDI, una trabajadora social de la Administración, una psicóloga, y una responsable de un servicio de vivienda concertada. Concretamente, las profesionales de entidades sociales cuentan con 
más de 20 años de experiencia en la gestión de residencia para PDI y en atención al colectivo e impulso de nuevos servicios.

- Grupo de Discusión de Familiares (en adelante GF). Se ha contado con la participación de cuatro familiares de personas que residen en una residencia de gestión pública y dos familiares de personas que residen en una vivienda gestionada por una asociación. Por último, también ha participado un familiar de una persona que se encontraba en lista de espera en el momento de la investigación.

\section{Motivos para solicitar plaza de residencia y buenas prácticas familiares}

En primer lugar, se analizan cuáles son los motivos principales para que las familias soliciten plaza de residencia para las PDI. En esta cuestión, las profesionales consultadas consideran que ha habido un cambio sustancial en la demanda de plaza de vivienda desde el modelo de concertación de los servicios que garantizan, además de la plaza, el transporte. En los años 80 o 90, cuando las plazas de residencia no eran ni eran concertadas ni subvencionadas, la atención residencial era para jóvenes y adultos del medio rural que las necesitaban para poder acceder a servicios de colegio o centro ocupacional. Llama la atención que desde los 10 años de edad ocupaban plaza, niños, jóvenes, adolescentes y adultos en la misma residencia.

Cuando los centros ocupacionales no estaban concertados en el medio rural nuestras asociaciones no tenían los recursos suficientes para pagar el transporte hasta Nájera o hasta Calahorra y no podían acceder al servicio. Una vez que se conciertan y que tienen garantizado ese servicio. (GP)

Desde la concertación en 2008, las plazas pasan de ser de semana a permanentes y para personas mayores de 18 años y de grados II y III de dependencia. Estos requisitos dejan fuera a las personas con discapacidad intelectual con grado I o sin grado, como se apunta en el debate en el que se observa que con anterioridad las PDI tenían acceso por el simple hecho de contar con un certificado de más del 33\% de discapacidad.

Ante la pregunta de cómo llegan las personas a la residencia, qué necesidades cubre, habría que decir que hay necesidades que quedan sin cubrir (...) personas que tienen padres mayores, orfandad, etc., no tienen plaza si tienen grado I o no tienen reconocido el grado. (GP)

Acerca de si las necesidades extensas de las personas que solicitan plaza es debido a un perfil de personas grandes dependientes, con muchas necesidades de apoyo extensas o generalizadas se observa que hay ya muchas personas en proceso de envejecimiento "con lo cual el deterioro cognitivo, el apoyo físico en todas las actividades es total" (GP). Una posible circunstancia que puede darse es que se dé una alta incidencia de personas con problemas de conducta, con trastornos mentales, debido al significativo porcentaje de personas que son usuarias de la Unidad de Salud Mental (USM) en los centros de atención diurna. Se analiza esta circunstancia en el perfil actual de las personas residentes y se constata que no es muy reseñable, aunque indican que: 
Los centros residenciales se están convirtiendo en un cajón desastre, el CAPDP (Centro de Atención a Personas con Discapacidad Psíquica), concretamente es un centro muy grande que, en su momento, como había plazas, se llenó. (GP)

Se da el caso de personas a las que no se les ha diagnosticado o, con el paso del tiempo y su deterioro, presentan trastornos de conducta y que actualmente permanecen en recursos residenciales para PDI. A estas personas, si no se puede prestar el apoyo que necesitan, se les derivará a otros recursos más específicos. Estos servicios de atención residencial para personas con el denominado "trastorno dual", que presentan discapacidad intelectual y trastorno mental, no existen en la comunidad autónoma de La Rioja y es una demanda del colectivo al gobierno regional ya que es otro perfil que se queda fuera de los recursos de vivienda.

En cuanto a la variable de exclusión social y otras necesidades de las familias, se dan casos de personas con un perfil socio familiar más complicado. Las últimas incorporaciones de jóvenes provienen de recursos de protección que deben dejar a los 18 años y solicitan plaza para personas con discapacidad si tienen grado II, ya que no hay recursos de alojamiento a partir de la mayoría de edad. Analizado el perfil actual de las personas que ocupan plaza de residencia, son personas con grados II o III de dependencia, con padres mayores o en situación de orfandad. La edad media de residentes es de 45-58 años.

En cuanto a los motivos individuales para solicitar plaza, es coincidente la difícil conciliación del trabajo y el apoyo a las PDI, ya que los recursos existentes no facilitan la permanencia en el hogar.

Al fallecer padres y no tener más hermanos, no hay facilidades para alternar trabajo con las perspectivas que tiene ella. Los acompañamientos fueron desapareciendo (no había monitores), El SAD (Servicio de Ayuda a Domicilio) fue insuficiente. (GF)

Mi hermana estamos en periodo de espera, es una situación dura (...), pero la situación familiar de la casa (....) tengo a los padres muy mayores, mi padre tiene 89 años, anda con un taca, mi madre con 85 ni se mueve, no anda. Yo ando enferma, tengo que ingresar bastantes veces, ha llegado un momento que no sé qué hacer con la situación... son tres personas... trabajo en el campo, pero ya ni puedo. (GF)

Uno de los mecanismos de entrada para ocupar plaza de residencia es ingreso urgente en caso de riesgo social acreditado, del que se pueden beneficiar las personas cuyas circunstancias familiares se pueden constatar que son graves o cuando es requerido por orden judicial. En estos casos las personas pasan a ocupar la primera plaza libre, por delante de cualquier otra persona. Esta posibilidad, aunque sean pocas las plazas disponibles en general en La Rioja, garantiza a las familias que, de darse una situación límite de gravedad la PDI, contase con un recurso residencial.

Yo tengo un primo. Mi tía está enferma y su padre se quedó en silla de ruedas. Mi primo tiene una discapacidad muy importante físicamente, entonces no se pudieron hacer cargo... fue de 
la noche a la mañana, le dieron una plaza (....) la situación era insostenible. Era nuestra salvación. (GF)

Tras analizar las casuísticas que presentan las familias y que han motivado la solicitud de plaza de residencia permanente se puede concluir que, en muchos de los casos, la primera opción de vivienda tras el fallecimiento de los padres es la casa del hermano tutor. Ante las dificultades de conciliación y la inexistencia de servicios de apoyo para permanecer en casa o de alternativas de vivienda, las PDI se ven abocadas a solicitar plaza de residencia permanente. Por otra parte, cuando los padres pasan de un rol de cuidadores principales de las PDI a ser perceptores de atención, por enfermedad o discapacidad sobrevenida, las PDI salen de la vivienda ante la dificultad de contar con recursos de apoyo suficientes.

En cuanto a los efectos en las relaciones sociales y familiares el ocupar una plaza en residencia, éstas van a depender de qué necesidad cubre el solicitar plaza de residencia, de la edad de la PDI y de sus familiares. Se observan casos de padres que planifican el futuro de sus hijos y toman la decisión del paso a ocupar plaza de residencia antes de que fallezcan. No obstante, cabe señalar el sentimiento de frustración que conlleva el hecho de no poder atender a las necesidades de apoyo de la PDI. Por ello, resultaría necesario generar medidas proactivas para que la familia no vea paralizada su actividad por ese sentimiento de culpa o que no sea un motivo de menos relación, por el malestar que se sienten al ver a su hijo en una residencia. En los casos de dificultades de convivencia por problemas de conducta de la PDI, las familias pueden sentir más alivio al pasar vivir fuera del hogar.

Si el motivo es que hay trastorno de conducta o problemas en casa hay un doble sentimiento, de alivio y respiro y sentimiento de culpa. (GP)

Cuando son los hermanos los que solicitan el ingreso, éstos desean que compartan, que estén con iguales. Se observa que el paso del núcleo de convivencia con padres mayores a un servicio residencial hace que la persona tenga experiencias positivas de relación. Se ha constatado que la vinculación con la familia cambia, la PDI pasa de estar con padres muy mayores, con un nivel de actividad muy limitado a estar en un entorno más activo. Esto provoca que la PDI no quiera ir a casa de forma frecuente.

En cuanto a la organización familiar aparecen diferencias, dependiendo de la flexibilidad del servicio en el que viven las PDI. Ante servicios en los que hay horarios muy concretos para realizar las visitas y para salir, la dificultad para establecer relaciones familiares satisfactorias se incrementa. Sin embargo, cuando en el servicio residencial hay una apertura a la familia, sin horarios prefijados, con la posibilidad de acudir sin previo aviso, sacar al familiar a comer cuando desee, poder entrar en su habitación e incluso comer con ella en su residencia, se nota una gran diferencia y las relaciones familiares se mantienen e incluso se aumentan.

Puedo llamar cuando yo quiera, ella me llama también. Pongo el horario que me va bien para recogerle. Subo cuando quiero a su habitación sin problemas, paso por ahí y entro a verle. (GF) 


\section{Reflexiones para la prevención de la institucionalización}

En algunas de las residencias de La Rioja se están dando buenas practicas que previenen la institucionalización. En cuanto a cómo hay que cuidar la gestión de la residencia para que se considere que favorece que las personas que ahí viven mantengan las relaciones con familiares y puedan establecer otras con personas de su entorno próximo, como es el barrio, se destaca la evolución que se ha dado. En la actualidad, se constata la existencia en la comunidad autónoma riojana de una vivienda abierta a familiares y amigos, con flexibilidad de horario para las visitas, pero también para que el residente entre o salga de su casa.

En nuestra residencia no hay horarios de visita, no hay restricciones de llamadas, no hay espacios. Se entiende que uno está en casa. (GP)

Si bien los modelos de atención residencial que se dan en La Rioja son contrapuestos, por diferentes motivos como el número de plazas o el tipo de gestión (pública o privada), se observa que hay un punto de inflexión que no se puede ni debe obviar. Aun así, las personas que residen en servicios con gestión más rígida, expresan sus deseos de flexibilidad. La transparencia, vista como la posibilidad de acceso a las instalaciones y poder participar del día a día, tranquiliza en gran medida a la familia y les resta el sentimiento de culpa. Del mismo modo, la flexibilidad favorece la relación.

Una buena práctica destacada es la de disponer de habitaciones individuales. Asimismo reflexionan acerca de que en las residencias de personas mayores las habitaciones son compartidas, lo que impide la intimidad de la persona y afecta a la calidad de vida del residente.

Una variable a tener en cuenta, es el caso de personas residentes que no disponen de una familia, bien porque pertenecen a un colectivo de exclusión, por ausencia de familia o porque no tienen una buena relación. Las medidas proactivas y estrategias que se están dando para mantener ese vínculo son fundamentalmente el apoyo a la PDI; con el objeto de que sean ellos quienes lideren su acercamiento, si así lo desean.

Depende del residente, si él no quiere no se hace nada, si él quiere se establecen alianzas, con unos con otros. Se ve si es positivo para él, pero si es que no, es que no. (GP)

En residencias con pocas plazas con la modalidad de apertura, el contacto con las familias es constante. Este aspecto resulta muy complicado cuando hay 100 residentes. Este es uno de los motivos por los que, desde entidades del Tercer Sector, se apuesta por recursos de vivienda con pocas plazas. En los recursos de vivienda centrados a priori en la atención a la persona residente, articular cómo se realiza el contacto con la familia es un tema delicado. El equilibrio en el tipo de relación del servicio con la familia debe ser cuidado desde el inicio de la misma aunque, para garantizar el apoyo adecuado a la PDI en el área de sus relaciones familiares, la apuesta que parece más adecuada es la de empoderar a la persona y apoyarle para que la demanda la haga la propia PDI, en el caso de que tenga la capacidad suficiente. 
Una buena práctica, valorada tanto por profesionales como por familiares, es aquella que fomenta las relaciones sociales significativas de las personas residentes. Se trata de un trabajo que se hace conscientemente, que se sustenta en el conocimiento de los gustos y preferencias de las personas y en el fomento de la participación social de las mismas. Esto, a su vez, genera oportunidades para que las PDI establezcan relaciones individuales con otras personas, sin discapacidad y con aficiones similares. Para dar este paso a la participación en la comunidad es clave el empoderamiento de las PDI, junto a un "entrenamiento, entrenamiento y entrenamiento" (GP). En paralelo, sería necesario implicar a las familias para que autoricen las salidas, motivar a los profesionales, formarles y trabajar en equipo para que el servicio sea flexible. Tanto familiares como profesionales coinciden en que es imprescindible participar en la comunidad, en el barrio, es decir: estar presentes. Asimismo, es fundamental que el equipo esté muy motivado y que tenga interiorizado el objetivo.

Hay que tener buenos principios, pero no te garantiza que vaya a ir bien. Hay que creérselo. Si esto no funciona da igual que vivas cuatro en un piso, estás institucionalizado. (GP)

En línea a lo anterior, indican que el voluntario tutelar favorece que las PDI tengan relaciones significativas. Éste surge ante la necesidad de prestar apoyo al área afectiva y emocional de las personas tuteladas por las fundaciones. Se trata de una figura voluntaria que consiste en personas que comparten gustos y aficiones con PDI tuteladas y que establecen un vínculo emocional. No obstante, cabe cuestionarse si estas buenas prácticas pueden ser utilizadas con personas con grandes necesidades de apoyo; debido a que la población con discapacidad está envejeciendo y que las residencias van a incrementar el límite de edad de permanencia. La respuesta más generalizada es que se trata de atender a los gustos y preferencias de las personas con más necesidades de apoyos y de prestar los que precise. Para ello, si es necesario, aumentan la ratio, generado la necesidad de flexibilizar el servicio y la actitud positiva hacia el modelo.

¿A nivel de relaciones? Igual, tú tienes las preferencias de las personas con grandes necesidades de apoyo, sus gustos, la persona te lo dice, hay un histórico... pero la distribución de los apoyos será diferente. Igual necesitas a tres personas, reorganizar el servicio, los recursos humanos para mantener todo eso, igual que mantienes la autonomía las relaciones hay que mantenerlas también. (GP)

Las personas que viven en una residencia desean mantener la libertad de toma de decisiones en pequeñas tareas del día a día como cuándo se asean, qué ven en la televisión, dónde se sientan a comer; o en asuntos tan importantes como con quién comparten habitación, en el caso de verse obligadas a hacerlo. También verbalizan el deseo de alcanzar cuotas de libertad para salir de la residencia con amigos, sin el apoyo de profesionales en gran grupo. Las familias tienen en cuenta el funcionamiento, la gestión y la estructura, ya que condiciona el tipo de servicio. Para favorecer las relaciones familiares, una necesidad de las familias es la de disponer de libertad de horarios para visitas y llamadas. El hecho de 
que la persona viva en un recurso residencial no debe impedir que las familias puedan compartir experiencias que son gratificantes para las PDI. En los centros de atención diurna se da la oportunidad a las familias de acudir a actividades del centro, en el caso de las residencias debería contarse con esa posibilidad.

Tan sencillo que tengan libertad dentro de sus necesidades para poder disfrutar lo que tienen en ese momento, el sitio, sus amigos... como si están en su casa. Salir de trabajar y poder tomarme un café con ella, a diario, que me llame y me diga estás en casa, pues voy a hacerte una visita o me voy contigo al cine. Sentir que me hacen partícipe en la vida de mi hermana. (GF)

En relación a las actividades en la comunidad, es imprescindible que exista libertad para participar en espacios normalizados, se debe fomentar desde la residencia para que tengan una vida activa, facilitando los apoyos necesarios, así se garantiza la relación con otras personas sin discapacidad.

Que les faciliten, que no les impidan hacer ese tipo de actividades. (GF)

A diferencia de los profesionales, en ocasiones las familias consideran difícil que personas jóvenes sin discapacidad establezcan relaciones de amistad con PDI. Éstos opinan que, a partir de una edad la sociedad no tiene en cuenta a las personas con discapacidad entre sus amistades. No obstante, si se dan las circunstancias de participación activa como ciudadanos en espacios inclusivos, las relaciones entre PDI y otras personas sin discapacidad podrían verse favorecidas.

\section{Conclusiones}

El presente estudio ha dado respuesta a los objetivos planteados y, además, supone un apoyo para la implantación de buenas prácticas en la gestión de futuros servicios residenciales para personas con discapacidad intelectual. Para poner en práctica servicios residenciales sin caer en la institucionalización; por una parte, se deben realizar prácticas que favorezcan el mantenimiento las relaciones familiares y que faciliten el establecimiento de relaciones sociales con otras personas sin discapacidad en entornos inclusivos. Por otra parte, las personas residentes deben ejercer su autodeterminación en el día a día de la vivienda, de manera práctica y real, tomando decisiones acerca de sus asuntos cotidianos.

Para atender las necesidades de bienestar emocional vinculadas a las relaciones personales, desde el momento del diseño de un recurso de residencia para personas con discapacidad, sería aconsejable mantener un enfoque sistémico de las necesidades de apoyo individual. Para que esta mirada sistémica en la atención en los servicios residenciales se lleve a la práctica, es fundamental el asesoramiento técnico de la figura de la trabajadora social. Se trata de una figura profesional que, en las entidades de atención a las PDI, ejerce el rol de apoyo a la familia, tiene presente las necesidades de éstas en su adaptación a los servicios, facilita la conciliación, etc. Del mismo modo, aporta el saber experto y el conocimiento de la realidad social y familiar de la persona en el momento de solicitar plaza 
(estado de salud de los miembros de la familia nuclear, dinámicas de relación, etc.) Todos estos elementos son importantes para planificar el apoyo al futuro residente con el objetivo de mantener o mejorar sus relaciones familiares y sociales. La familia es el sistema natural para proporcionar apoyo emocional a sus miembros y el paso a residencia no debe marcar una distancia con la PDI.

Para descentralizar la mirada y ampliar la atención de las personas residentes hacia su contexto social y familiar anterior a su ingreso, con el objetivo de favorecer relaciones naturales futuras, resulta necesario anticipar mecanismos de gestión flexibles y adaptados a las necesidades, deseos y expectativas de cada persona que ocupa plaza de residencia. Asimismo, se considera imprescindible un cambio de modelo que garantice el bienestar emocional de las personas residentes. Del mismo modo, según se ha constatado, resultaría clave generar un tejido social inclusivo, el cual implica ir más allá de un recurso concreto. Por ello, una manera de intervenir podría ser desde la perspectiva del trabajo social comunitario, con actuaciones de sensibilización que provoquen oportunidades de participación en actividades normalizadas con las personas con discapacidad.

Las personas con discapacidad tienen unos derechos que se pueden alcanzar desde una serie de apoyos dirigidos a facilitar que vivan de la manera más adecuada posible en consonancia con sus necesidades. La disciplina del Trabajo Social tiene un conjunto de herramientas, tanto teóricas como prácticas, que permiten apoyar a las personas con discapacidad intelectual e intervenir desde individual y familiar hasta lo grupal y comunitario; ofreciendo calidad y favoreciendo que estas personas mejoren en las diferentes dimensiones de su vida.

\section{Bibliografía}

Huete García, A., Huete García, M. Á., y Martín GómeZ, Á. (2015). Institucionalización de las personas con discapacidad en España. Observatorio estatal de la discapacidad.

Instituto DE MAYORES y SERvicios SOCIALES, IMSERSO (2006). Institucionalización y dependencia.

Instituto NaCional De Estadística, INE (2008) Encuesta sobre Discapacidad, Autonomía personal y situaciones de Dependencia (EDAD 2008).

ORGANIZACIÓN MUNDIAL DE LA SALUD, OMS (2001). Clasificación Internacional del Funcionamiento, la Discapacidad y de la Salud (CIF). Ginebra, Organización Mundial de la Salud.

ORGANIZACIÓN NACIONES UNIDAS, ONU (2006). Convención Internacional sobre los Derechos de las Personas con Discapacidad, Protocolo Facultativo.

ORGanización NACIONES UnidAS, ONU. Comité sobre los Derechos de las Personas con Discapacidad (2019). Observaciones finales sobre los informes periódicos segundo y tercero combinados de España del Comité sobre los Derechos de las Personas con Discapacidad (p. 14).

SCHALOCK, R.L. (2009). La nueva definición de discapacidad intelectual, apoyos individuales y resultados personales [The New Definition of Intellectual Disability, Individual Supports, and Personal Outcomes]. Siglo Cero, 229, pp. 22-39. Recuperado de: TAMARIT, J. (2015). "La transformación de los servicios hacia la calidad de vida. una 
iniciativa de innovación social de FEAPS". Siglo Cero, 46 (3), pp. 47-71. https://doi.org/10.14201/scero20154634771.

VERDUGO, M.A. (2010). "Últimos avances en el enfoque y concepción de las personas con discapacidad intelectual". Siglo Cero, 236, pp. 7-21.

Verdugo, M. A., Schalock, R. L., Thompson, J., y GuilléN, V. (2011). Discapacidad intelectual: definición, clasificación y sistemas de apoyo. Madrid, Alianza. 